Sharif University of Technology
Scientia Iranica
Transactions E: Industrial Engineering
IRAN I I IA

\title{
A novel two-stage stochastic model for supply chain network design under uncertainty
}

\author{
M. Mohajer Tabrizi ${ }^{a}$, B. Karimi ${ }^{a, *}$ and S.A. Mirhassani ${ }^{b}$ \\ a. Department of Industrial Engineering \& Management Systems, AmirKabir University of Technology, Tehran, Iran. \\ b. Department of Mathematics \& Computer Science, AmirKabir University of Technology, Tehran, Iran. \\ Received 7 March 2015; received in revised form 23 August 2015; accepted 12 October 2015
}

\author{
KEYWORDS \\ Supply chain network \\ design; \\ Strategic and \\ non-strategic items; \\ Supplier contracts; \\ Uncertainty; \\ Sample average \\ approximation; \\ Two-stage stochastic \\ programming; \\ Benders \\ decomposition.
}

\begin{abstract}
This paper addresses the problem of designing a supply chain network consisting of suppliers, manufacturers, warehouses, and customers in which all manufacturers belong to a single owner. All players in this chain are performing under uncertainty. The single product of this supply chain needs one strategic and one non-strategic part for its final assembly. To hedge against uncertainty in supply and demand, the manufacturers tend to take part in a set of suppliers through a portfolio of contracts, and unmet demand will be satisfied by purchasing from spot market with stochastic prices. In addition, demands, supply capacities, and warehouse capacities are stochastic as well, and the problem has been modeled as a two-stage stochastic program with recourse. Then, a hybrid solution strategy based on sample average approximation and accelerated Benders decomposition is proposed to tackle the problem. The proposed strategy is able to obtain good quality solutions for a large number of scenarios and within an acceptable time interval. Computational results show the effectiveness of the stochastic model as compared to its deterministic counterpart.

(C) 2016 Sharif University of Technology. All rights reserved.
\end{abstract}

\section{Introduction}

A supply chain is a system of suppliers, manufacturers, distributors, retailers, and customers. In this system, materials move from suppliers to customers and information flows in both directions [1]. A crucial component in the tactical phase of decisions in a supply chain is the way in which the manufacturer sets its raw material routes for stable supply of products to customers. These connection routes are usually constructed by contracts between manufacturer and suppliers. Contracts become more and more important to manufacturers considering the fact that many parameters in a decision-making are not certainly known prior to their realization in the real world. Many contracts have been designed so far to be used by industry in different situations such as long-term,

*. Corresponding author. Tel.: +982164545374

E-mail address: b.karimi@aut.ac.ir (B. Karimi) option, etc. In a long-term contract, the supplier guarantees the manufacturer to send a pre-specified amount of raw material at an agreed-upon price in a pre-determined period of time. A big disadvantage of this contract relates to the inventory holding costs. In option contracts, however, supplier agrees to send the raw material by a pre-determined time just at an agreed-upon level. In this contract, manufacturer pays a fraction of the price for all parts at the time contract is signed (premium fee) and will be charged for each unit it buys (execution fee). Although option contract may seem to be able to hedge manufacturer against risks, the total price is more in option than long-term contract. Thus, a company like HP used a portfolio of these contracts successfully to avoid the disadvantages of each contract [1].

Contracts named so far are usually used for nonstrategic items [1]. Kraljic argued that different supply strategies should be used for different items [2]. He named parts with few number of suppliers and great 
impact on profit as strategic items and parts with many suppliers and little profit imapct as non-critical items. Several types of contracts have been suggested for strategic items: buyback, payback, revenue sharing, etc. Since the supplier should prepare capacity to ensure its supply power before the real demand for strategic items, it is prevalent in many industries that manufacturers pass inflated demand predictions to suppliers. This phenomenon has side effects on both sides; sometimes suppliers are left with unused capacities and sometimes manufacturers cannot reply to their demand as for Boeing orders in 1997 [3]. To resolve this problem, some researchers have proposed special kinds of contracts, namely capacity reservation contract and advanced purchase contract. In the latter, a supplier charges a manufacturer an agreed price before demand is realized and charges a different price afterwards. In the former, supplier presents a list of capacities with corresponding prices. These kinds of contracts will enable suppliers to prevent manufacturers from inflating their orders [4].

In this paper, a supply chain network design problem in which a sole manufacturer sells a single product to a set of customers is considered. The product is assumed to need a strategic part along with a non-strategic part for completion. The manufacturer is faced with a stochastic demand and stochastic spot prices for its non-strategic part. To hedge against uncertainty, the manufacturer tends to select a portfolio of suppliers with different contracts. To avoid inflated orders, the supplier of strategic item has presented a capacity reservation contract. The question is: What is the right supply chain design for this manufacturer and how the portfolio of contracts should be constructed? The remainder of this paper is as follows. In the next section, a review of the literature is conducted. In Section 3, problem description is presented. In Section 4, the solution strategy is described. Section 5 presents the results of computational studies. Finally, this paper is concluded in Section 6 .

\section{Literature review}

Supply Chain Network Design (SCND) has attracted many researchers. Thanh et al. [5] addressed the problem of designing and planning a multi-period, multi-echelon, multi-commodity supply chain whereby each product has a bill of materials and all parameters are deterministic. Each plant and warehouse has limited capacity and lower and upper bounds on the utilization level. The comprehensive model developed there captures many modeling issues including opening, closing or expanding facilities, supplier selection and planning the distribution flow, etc. Nagurney [6] considered the problem of designing and redesigning a supply chain from a different modeling approach, i.e., variational inequalities. Badri et al. [7] developed a new mathematical model for multi-echelon, multicommodity dynamic SCND considering expansions of the supply chain according to cumulative net profits and fund supplied by external sources in contrast with common approach that expansion is restricted to a predetermined fund or to a fixed number of facilities. Correia et al. [8] presented two new models for a twoechelon dynamic system. Main decisions are location of new facilities, installation of warehouses, and distribution centers. Decisions are bound to a given budget and different product families are considered, while the first model is a cost minimization and the second one is a profit maximization one.

In another stream of research in the field of SCND, researchers have tried to incorporate uncertainty in their models. Santoso et al. [9] addressed a singleperiod, single-commodity network design problem in which the two-stage model determines which facilities should be opened, in addition to the technology of each facility and who the suppliers for each facility should be. They also proposed a hybrid of Benders decomposition and SAA as the solution approach [10]. Their paper does not consider any strategic component into account. Tiwari et al. [11] proposed a novel model for SCND with cost minimization objective satisfying uncertain demands at a specified service level. Pan and Nagi [12] considered a SCND with the new feature of considering emerging new markets while demand is uncertain in an agile manufacturing setting. Main decisions are: selection of facilities, alliance among different facilities, production and distribution. Georgiadis et al. [13] proposed a new mathematical model for SCND with multiple products flowing in the network in multiple time periods. The model considers time-dependent uncertainties in demand. Rajgopal et al. [14] proposed a new two-stage stochastic model for the design and operation of remnant industry, motivated by metal industry. The authors proposed a modified version of the L-shaped method to solve it. Sawik [15] looked for a portfolio of suppliers in a make-to-order supply chain. Selection criteria in his work are price, quality, and due date reliability. In addition, the mere uncertain parameter is the production rate of suppliers. $\mathrm{Li}$ and Zabinsky [16] proposed a two-stage and a chance constraint model for a supplier selection problem with uncertainties in demand and supply. The aim of the paper is to find the minimum number of suppliers, in addition to the order sizes from suppliers. Lin and Wang [17] studied the supply chain network design under supply and demand disruptions, while most of the past researchers only studied the demand-side disruption. They studied the supply chain network design under supply and demand uncertainty with embedded supply chain disruption mitigation strategy and manufactur- 
ing postponement with downward substitution. Pishvaee and Razmi [18] proposed a multi-objective fuzzy mathematical programming model for designing an environmental supply chain under inherent uncertainty of input data. Goetschalcks et al. [19] considered a multi-SCND problem with uncertainties in demand, capacities of suppliers, manufacturers, warehouses, and fixed location costs. Uncertainty is modeled as a set of scenarios. The risk of the system is modeled as the two-sided standard deviation of the profits of the various scenarios. Tabrizi and Razmi [20] considered a SCND problem in a multi-commodity, multi-stage, multi-capacity, and multi-source mode with sources of uncertainty in supply, demand, and processing sides.

Pishvaee et al. [21] studied the impact of social and environmental issues on supply chain network design. They used a multi-objective possibilistic programming approach to model a real case in the medical supply chain. Multiple conflicting objectives are converted to a single objective by means of a posteriori fuzzy approach, and an accelerated version of Benders is applied to solve the model.

Yolmeh and Salehi [22] proposed a new model for integrated supply chain network design and assembly line balancing under demand uncertainty. Their model determines the location of manufacturers and assemblers, balances assembly lines, and presents the required flow of products in the network. In the study of Wang and Lee [23], the authors addressed a capacitated location-allocation problem for a multi-echelon supply chain with stochastic demand variability. The authors proposed a revised version of the well-known ant algorithm.

Melo et al. [24], in their review paper, concluded that there is a gap in the literature related to consideration of decisions in a supply chain such as transportation modes, routing, and purchasing (contracts). Paksoy et al. [25] proposed a novel model consisting of multiple suppliers, manufacturers, distribution. To hedge against these uncertainties, the manufacturers use option contracts. The model answers the question of with which suppliers these contracts are signed and for which quality level. Xu and Nozick [26] considered a multi-period single-commodity supply chain with demand as the only uncertain parameter. Manufacturers belong to a single owner. Their model tries to find the right amount of raw material to purchase from each supplier using long-term and option contracts. In addition, an L-shape algorithm has been used to address the problem. Feng et al. [27] proposed a twostage stochastic programming model for coordinated contract design in a supply chain. The article considers a multi-site manufacturer which signs contracts with both suppliers and customers. Table 1 summarizes the main features of our research in contrast with abovementioned literature with respect to design and modeling features.

\section{Problem description}

Consider a supply chain network with four echelons consisting of suppliers, manufacturers, warehouses, and customers. In the supply chain under consideration, all manufacturers belong to a single owner. These manufacturers produce a single product and supply customers in different market segments through external distribution warehouses. The mere product of the supply chain is itself comprised of two different parts. The first part is a non-strategic component and could be supplied from a set of suppliers. The second part is a strategic component in the sense that it has only an exclusive supplier, and this supplier needs to build capacity before the production season, and thus, it requires its customers to reveal their production plan. However, the sole supplier of strategic component has designed a capacity reservation contract to ensure uninflated submit of orders from manufacturers. The supplier of strategic component presents a list of capacities linked with prices and the manufacturer signals its real demand by ordering a specific level. In real world, many of the parameters for decisionmaking are not known with certainty, therefore, to hedge against uncertainties from supply and demand, these manufacturers want to construct a portfolio of contracts with suppliers comprised of long-term and option contracts. In this study, capacity of nonstrategic suppliers, storage capacity of warehouses, as well as demand for different markets have been considered uncertain. Also, the manufacturers would react to unmet demand from customers with spot purchasing. The objective of the model is to minimize the total costs of engaging with suppliers, contracting with suppliers, and operating the supply chain. Main decisions in this model include: whether to engage with a non-strategic supplier or not; the amount of strategic component to order from its sole supplier; the amount of long-term contract with each supplier; and the appropriate option level with each supplier. The architecture of the supply chain studied in this paper is illustrated in Figure 1.

\section{Modeling assumptions}

Main assumptions used in the model of this research are as follows:

1. The location and number of suppliers, manufacturing plants, warehouses, and customers are known;

2. The suppliers of non-strategic parts propose a price for long-term contracts and an option list for pricevolume pairs; 
Table 1. Comparison of literature with the current study from design and modeling aspects.

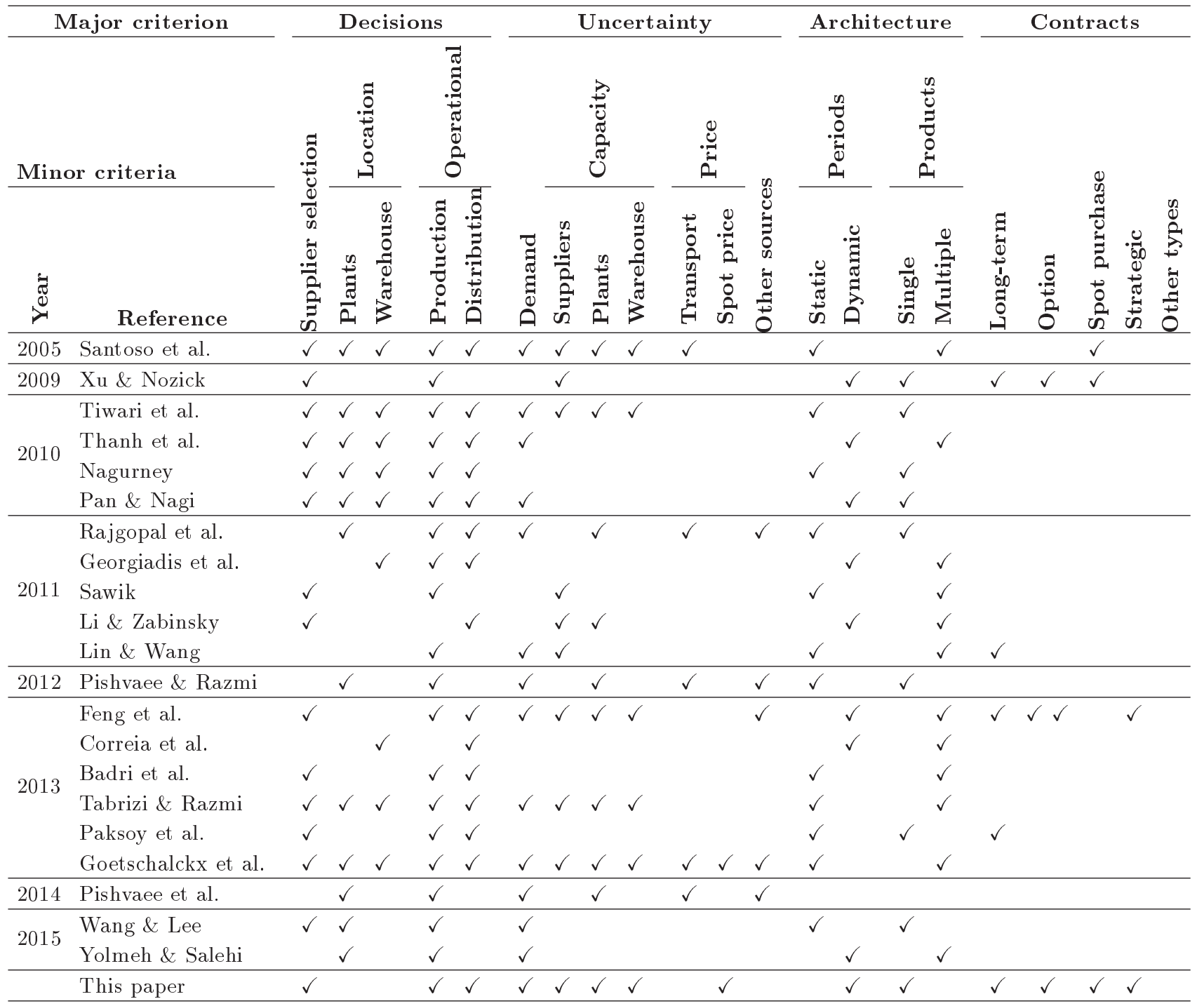

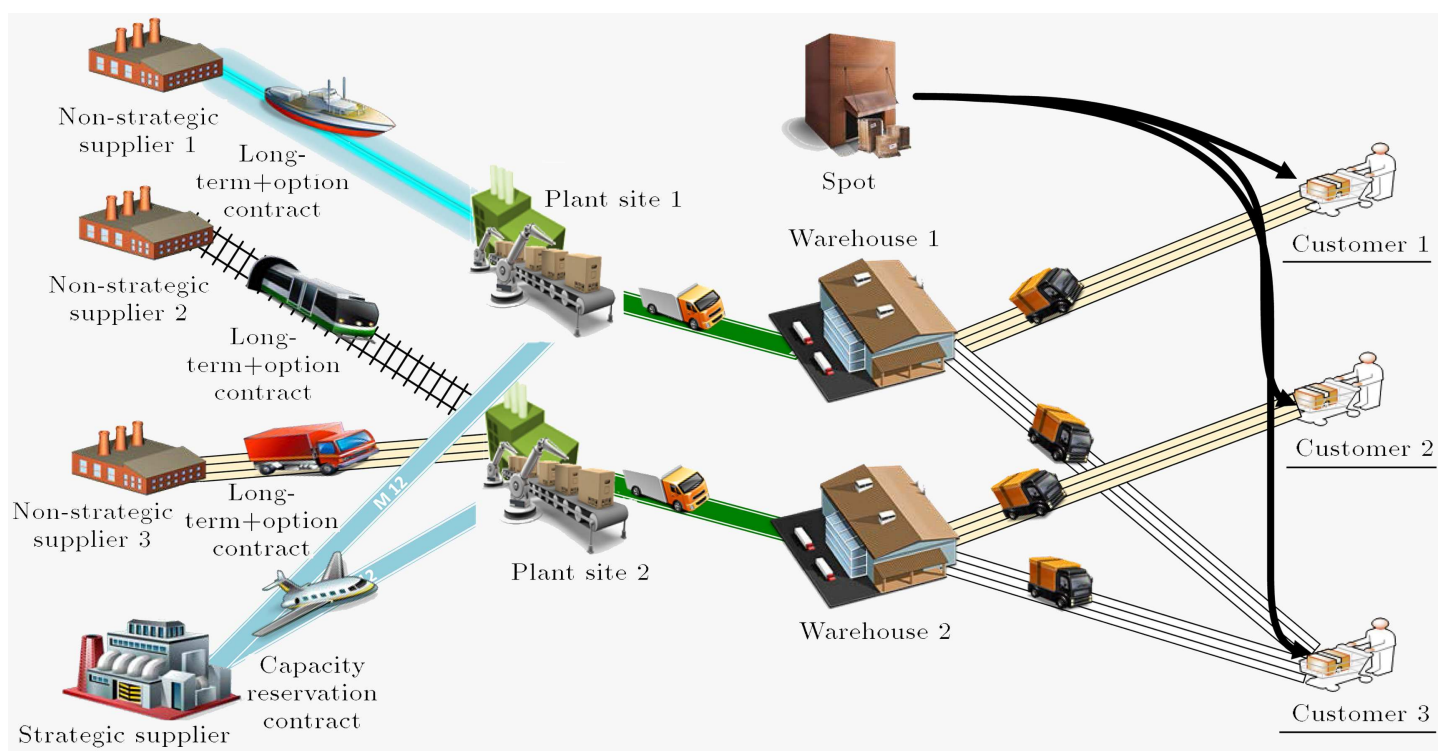

Figure 1. The supply chain configuration for this paper. 
3. It is assumed that unmet demand will be outsourced from spot market;

4. A scenario based approach is used to model uncertainty.

The modeling approach utilized in this paper is twostage stochastic programming with recourse. The reason for using this approach is that contractual decisions are integrated into the decisions regarding the distribution of components and goods, and decisions regarding contracts are usually made before real situations are realized. Suppose that the vector $y$ is the set of the first stage variables with cost vector $d$, while $x$ is the vector of the second stage variables with cost $q$. Also, assume that $\xi$ denotes the uncertain parameter vector:

$$
\min _{y \in Y} \mathbb{E}(\mathcal{F}(y, \xi))=d^{T} y+\mathbb{E}(\mathcal{Q}(y, \xi)),
$$

where:

$$
\begin{aligned}
& \mathcal{Q}\left(y, \xi^{n}\right)=\min _{x_{n}} q_{n}^{T} x_{n}, \\
& \text { s.t: } W_{n} x_{n}=h_{n}-T_{n} y, \quad x_{n} \geq 0
\end{aligned}
$$

Problem in Eq. (1) is called the "first stage" and problem in Eq. (2) is called the "second stage" problem in the literature. The notations used in the mathematical model are as follows:

\section{Sets \& indices}

$C \quad$ Set of customers indexed by $c$

$P \quad$ Set of plants indexed by $p$

$S \quad$ Set of the non-strategic suppliers indexed by $s$

$W \quad$ Set of warehouses indexed by $w$

I Set of options for the non-strategic parts indexed by $i$

$K \quad$ Set of purchasing intervals for the non-strategic parts in long-term contract indexed by $k$

$J \quad$ Set of capacity reservation possibilities for the strategic parts indexed by $j$

$T \quad$ Set of time periods indexed by $t$

$N \quad$ Set of scenarios indexed by $n$

\section{Parameter}

Fst $_{j} \quad$ Fixed cost of reserving capacity $j$ for the strategic item

$F_{o} \quad$ Premium cost of buying option $i$ for the non-strategic items from supplier $s$

$\mathrm{Fl}_{k s} \quad$ Fixed cost of purchasing long-term contract level $k$ from supplier $s$

$F c_{s} \quad$ Fixed cost to engage with non-strategic supplier $s$
Tst $t_{p} \quad$ Transportation cost from the strategic supplier to plant $p$

$T s p_{s p} \quad$ Transportation cost from the non-strategic supplier $s$ to plant $p$

$T p w_{p w} \quad$ Transportation cost from plant $p$ to warehouse $w$

$T w c_{w c} \quad$ Transportation cost from warehouse $w$ to customer $c$

$C s_{s t n} \quad$ Total capacity of non-strategic supplier $s$ in period $t$ for scenario $n$

$C w_{w t n} \quad$ Capacity of warehouse $w$ in period $t$ for scenario $n$

est $t_{j} \quad$ Variable cost for each strategic part purchased

$e_{s} \quad$ Exercise cost of using option contract from supplier $s$

$g t_{j} \quad$ Available strategic items in each week under capacity reservation $j$

$g o_{i} \quad$ Available items in each week under option $i$ from each supplier

$g o_{k} \quad$ Available items in each week under long-term level $k$ from each supplier

$P_{c t n} \quad$ Spot price of final product to supply customer $c$ in period $t$ for scenario $n$

$d_{c t n} \quad$ Demand from customer $c$ in period $t$ for scenario $n$

$h_{w} \quad$ Holding cost of each unit of inventory in unit time in warehouse $w$

$\beta \quad$ Total number of available suppliers for the non-strategic items

\section{Variables}

yst $_{j} \quad$ Equal to 1 if level $j$ for capacity of strategic is reserved, 0 otherwise

yo $\quad$ Equal to 1 if option level $i$ for non-strategic item from supplier $s$ is bought, 0 otherwise

$y l_{k s} \quad$ Equal to 1 if long-term level $k$ for non-strategic item from supplier $s$ is bought, 0 otherwise

$y c_{s} \quad$ Equal to 1 if the manufacturers decide to engage with supplier $s, 0$ otherwise

$x_{s t} t_{p t n} \quad$ Amount of strategic part sent to plant $p$ in period $t$ for scenario $n$

$x o_{s p t n} \quad$ Amount sent from supplier $s$ to plant $p$ in period $t$ for scenario $n$ - option contract

$x l_{s p t n} \quad$ Amount sent from supplier $s$ to plant $p$ in period $t$ for scenario $n$ - long-term contract

$x p w_{p w t n} \quad$ Amount sent from plant $p$ to warehouse $w$ in period $t$ for scenario $n$

$x w c_{w c t n} \quad$ Amount sent from warehouse $w$ to customer $c$ in period $t$ for scenario $n$ 
$z_{c t n} \quad$ Unmet demand of customer $c$ in period $t$ for scenario $n$

$\operatorname{Inv}_{w t n}$ Inventory level at warehouse $w$ in period $t$ for scenario $n$

The two-stage model for our problem is then as follows:

First stage problem:

$\min _{y}:$

$$
\begin{aligned}
\sum_{s} F c_{s} y c_{s} & +\sum_{j} F s t_{j} y s t_{j}+\sum_{i, s} F o_{i s} y o_{i s} \\
& +\sum_{k, s} F l_{k s} y l_{k s}+\mathbb{E}(\mathcal{Q}(y, \xi)),
\end{aligned}
$$

s.t.:

$$
\begin{aligned}
& \sum_{j} y s t_{j} \leq 1, \\
& \sum_{i} y o_{i s} \leq y c_{s} \quad \forall s \in S, \\
& \sum_{s} y c_{s} \leq \beta, \\
& \sum_{k} y l_{k s} \leq y c_{s} \quad \forall s \in S, \\
& y s t_{j}, y o_{i s}, y c_{s}, y l_{k s} \in\{0,1\},
\end{aligned}
$$

where $\mathcal{Q}(y, \xi)$ is as follows:

$$
\begin{aligned}
& \min _{x, z} q_{n}^{T} x_{n}=\sum_{p, t, n}\left(e s t+T s t_{p}\right) x s t_{p t n} \\
& \quad+\sum_{s, p, t, n} T s p_{s p} x l_{s p t n}+\sum_{s, p, t, n}\left(e o_{s}+T s p_{s p}\right) x o_{s p t n} \\
& \quad+\sum_{p, w, t, n} T p w_{p w} x p w_{p w t n}+\sum_{w, c, t, n} T w c_{w c} x w c_{w c t n} \\
& \quad+\sum_{c, t, n} P_{c t n} z_{c t n}+\frac{1}{T} \sum_{w, t, n} h_{w} \operatorname{Inv}_{w t n}, \\
& \sum_{p}\left(x l_{s p t n}+x o_{s p t n}\right) \quad \leq C s_{s t n} y c_{s} \\
& \forall s \in S_{i} t \in T, n \in N, \\
& \sum_{p} x o_{s p t n} \leq \sum_{i} g o_{i} y o_{i s} \quad \forall s \in S, t \in T, n \in N, \quad(2-2) \\
& \sum_{p} x s t_{p t n} \leq \sum_{j} g s t_{j} y s t_{j}
\end{aligned} \quad \forall t \in T, n \in N, \quad(2-3)
$$

$$
\begin{aligned}
& \sum_{p} x l_{s p t n} \leq \sum_{k} g l_{k} y l_{k s} \quad \forall s \in S, t \in T, n \in N, \\
& \forall p \in P, t \in T, n \in N \quad \forall p \in P, t \in T, n \in N, \\
& x s t_{p t n}=\sum_{w} x p w_{p w t n} \quad \forall p \in P, t \in T, n \in N,(2-2) \\
& \sum_{p} x p w_{p w t n} \leq C w_{w t n} \quad \forall w \in W, t \in T, n \in N,(2-7) \\
& \operatorname{Inv}_{w t+1 n}=\operatorname{Inv}_{w t n}+\sum_{p} x p w_{p w t n}-\sum_{c} x w c_{w c t n}, \\
& \forall w \in W, t \in T, n \in N, \\
& \sum_{w} x w c_{w c t n}+z_{c t n} \geq d_{c t n} \quad \forall c \in C, t \in T, n \in N,(2 \\
& \operatorname{Inv}_{w 0 n}=0 \\
& x s t_{p t n}, x o_{s p t n}, x l_{s p t n}, x p w_{p w t n}, x w c_{w c t n}, z_{c t n} \geq 0 .
\end{aligned}
$$

In the first stage problem, the objective is the minimization of the sum of fixed costs to establish the relationship and also to choose contract levels for all types of contracts, that is, strategic, option, and longterm. Constraints for this problem are of logical type. Constraint (1-1) ensures that just one of the capacity levels from strategic supplier should be reserved. Constraints (1-2) and (1-4) do the same job for option and long-term contracts while they also state that these contracts with non-strategic suppliers are chosen if there exists a relationship with them. Constraint (1-3) enforces the number of suppliers to be less than a certain number. In the second stage, the objective is comprised of four cost categories: cost of transportation of goods from one node to another in the chain, cost of using reserved capacities for strategic items and exercising options purchased, cost of shortage, and inventory holding cost at warehouses. Constraint (2-1) states that the total items bought from a supplier under long-term and option contracts should not exceed its total capacity in different time periods and scenarios, provided that the supplier is engaged with the manufacturers. Constraints (2-2) to (2-4) ensure that the amount the manufacturers purchase, with option, strategic, or long-term contracts, respectively, does not exceed the reserved capacity. Constraints (2$5),(2-6)$, and (2-8) are flow reservation constraints. Constraints (2-5) and (2-6) guarantee that the total raw materials (non-strategic and strategic) that enter a plant are equal to the finished products flow from this plant to all warehouses. Constraint (2-8) states that inventory at each warehouse in each time period, and 
scenario equals the inventory level in last period; plus, the products that enter the warehouse in the period minus the products that leave it. Constraint (2-7) is the capacity constraint for warehouses. Constraint (2-9) is the demand satisfaction constraint. Constraint (2-10) is based on the assumption that there is no inventory at any warehouse in the beginning of the planning horizon. Finally, Constraints (2-11) are non-negativity Constraints for the decision variables.

\section{Solution procedure}

In this section, the solution strategy is illustrated. Since this model is a two-stage model, the first idea is to use the L-shaped algorithm proposed by Van Slyke and Wets [28] to address the solution. However, since the number of scenarios is large in real instances, the problem of size grows astronomically. To illustrate the magnitude to which this matters, the example by Santoso et al. [9] is studied. Consider a supply chain with 50 facilities, which is ordinary for realistic cases, and that each facility has just one uncertain parameter. Moreover, suppose that this uncertain parameter has only three scenarios. In this situation, one may have to deal with $3^{50} \cong 7 \times 10^{23}$ scenarios, which are far more than the ability of the current technology to handle it. Moreover, our problem has a multi-period structure that will complicate the solving procedure of the problem with standard techniques such as L-shaped method. Therefore, we adopted SAA to handle the large number of scenarios. This procedure has its roots in Monte Carlo simulation and sampling in statistics. In each stage of the procedure, a smaller sample of the real problem has to be dealt with. However, since a tactical decision should be tackled, it still takes too much time to solve the problem with good quality using standard optimization soft wares. Therefore, Benders decomposition, suitable for the case of this paper, is proposed to solve the resulting MIPs in a reasonable time. However, as Benders decomposition in its standard format has a slow convergence rate, it is proposed in the following to use a mix of acceleration techniques to enable satisfactory results. In following subsections, first, the procedure of SAA and then Benders decomposition are illustrated. Subsequently, the acceleration techniques that have been used in this paper will be described.

\subsection{Sample average approximation}

In a stochastic program like the one stated in Eq. (1), the main difficulty arises in the calculation of expected value, since the function $\mathcal{Q}(y, \xi)$ is not explicitly at hand and in a closed analytical form. Even if it is accessible, computing such an expectation consists of multiple integrals or solving too many linear programs. To avoid these difficulties, a good idea is to use the sample average statistic instead of the original expectation. To do this job, Shapiro and Homemde-Melo [29] proposed to perform $M$ independent experiments. In each experiment, one must take $N$ independent samples from the main problem. In fact, each sample consists of solving "linear program 3" called "Sample Average Problem (SAP)".

$$
\hat{l}_{m}=\min \frac{1}{N} \sum_{n=1}^{N}\left(d^{T} y+Q\left(y, \xi_{n}^{m}\right)\right) .
$$

In this paper, the optimal solution to this problem is called $\hat{y}_{m}$, and SAP looks like the following (Eqs. (3-0) to $(3-15))$ :

$$
\begin{aligned}
& \hat{l}_{m}=\min _{x, y, z} \\
& \frac{1}{N}\left(\sum_{s} F c_{s} y c_{s}+\sum_{j} F s t_{j} y s t_{j}+\sum_{i, s} F o_{i s} y o_{i s}\right. \\
& \quad+\sum_{k, s} F l_{k s} y l_{k s}+\sum_{p, t, n=1}^{N}\left(e s t+T s t_{p}\right) x s t_{p t n} \\
& \quad+\sum_{s, p, t, n=1}^{N} T s p_{s p} x l_{s p t n}+\sum_{s, p, t, n=1}^{N}\left(e o_{s}+T s p_{s p}\right) x o_{s p t n} \\
& \quad+\sum_{p, w, t, n=1}^{N} T p w_{p w} x p w_{p w t n}+\sum_{w, c, t, n=1}^{N} T w c_{w c} x w c_{w c t n} \\
& \left.\quad+\sum_{s, t, n=1}^{N} P_{c t n} z_{c t n}+\frac{1}{T} \sum_{w, t, n=1}^{N} h_{w} \operatorname{Inv}_{w t n}\right) \\
& \sum_{i} y o_{s p t n} \leq \sum_{i} g o_{i} y o_{i s} \\
& \sum_{j} y s t_{j} \leq 1, \\
& \forall c_{s s} \leq y c_{s}
\end{aligned}
$$$$
\forall s \in S, t \in T, n \in N,
$$ 


$$
\begin{aligned}
& \sum_{p} x s t_{p t n} \leq \sum_{j} g s t_{j} y s t_{j} \\
& \forall t \in T, n \in N, \\
& \sum_{p} x l_{s p t n} \leq \sum_{k} g l_{k} y l_{k s} \\
& \forall s \in S, t \in T, n \in N, \\
& \sum_{s}\left(x l_{s p t n}+x o_{s p t n}\right)=\sum_{w} x p w_{p w t n} \\
& \forall p \in P, t \in T, n \in N, \\
& x s t_{p t n}=\sum_{w} x p w_{p w t n} \\
& \forall p \in P, t \in T, n \in N, \\
& \sum_{p} x p w_{p w t n} \leq C w_{w t n} \\
& \forall w \in W, t \in T, n \in N, \\
& \operatorname{Inv}_{w t+1 n}=\operatorname{Inv}_{w t n}+\sum_{p} x p w_{p w t n}-\sum_{c} x w c_{w c t n} \\
& \forall w \in W, t \in T, n \in N, \\
& \sum_{w} x w c_{w c t n}+z_{c t n} \geq d_{c t n}, \\
& \forall c \in C, t \in T, n \in N, \\
& \operatorname{Inv}_{w 0 n}=0 \quad \forall w \in W, n \in N, \\
& x s t_{p t n}, x o_{s p t n}, x l_{s p t n}, x p w_{p w t n}, x w c_{w c t n}, z_{c t n} \\
& \geq 0, y s t_{j}, y o_{i s}, y c_{s}, y l_{k s} \in\{0,1\} .
\end{aligned}
$$

Then, taking an average over $\hat{l}_{m}$ s gives a lower bound on the optimal value, i.e. $\bar{l}$. The variance of this estimator is calculated as in Eq. (4):

$$
s_{l}^{2}=\frac{1}{M(M-1)} \sum_{m=1}^{M}\left(\hat{l}_{m}-\bar{l}\right)^{2} .
$$

Then, to obtain a good quality solution, an upper bound should also be derived from the problem. To do so, a feasible first-stage solution should be used such as one of $\hat{y}_{m} \mathrm{~s}$ obtained in the last step (this feasible first-stage solution is named $\bar{y}$ from now on). Then, a new sample will be drawn with size $N^{\prime}$ and compute Eq. (5) to obtain the sample upper bound, i.e. $\bar{u}$.

$$
\bar{u}=\frac{1}{N^{\prime}} \sum_{n=1}^{N^{\prime}}\left(d^{T} \bar{y}+Q\left(\bar{y}, \xi_{n}\right)\right) .
$$

Note that in the above equations, we are solving $N$ single-scenario LPs $Q\left(\bar{y}, \xi_{n}\right)$ as exhibited below (from Eqs. (5-0) to (5-14)) which are easy to handle. Thus, since the accuracy of the sample upper bound increases with the increase in $N^{\prime}$, we may use a much larger sample size than the one used as $N$. The suggestion is about 1000 compared with about 30 or 60 for $N$.

$$
\begin{aligned}
& \mathcal{Q}\left(\bar{y}, \xi_{n=q}\right)=\min _{x, z}: \\
& \sum_{p, t}\left(e s t+T s t_{p}\right) x s t_{p t q}+\sum_{s, p, t} T s p_{s p} x l_{s p t q} \\
& +\sum_{s, p, t}\left(e o_{s}+T s p_{s p}\right) x o_{s p t q}+\sum_{p, w, t} T p w_{p w} x p w_{p w t q} \\
& +\sum_{w, c, t} T w c_{w c} x w c_{w c t q}+\sum_{c, t} P_{c t q} z_{c t q} \\
& +\frac{1}{T} \sum_{w, t} h_{w} \operatorname{Inv}_{w t q} \\
& \sum_{j} y s t_{j} \leq 1 \\
& \sum_{i} y o_{i s} \leq y c_{s} \quad \forall s \in S, \\
& \sum_{s} y c_{s} \leq \beta \\
& \sum_{k} y l_{k s} \leq y c_{s} \quad \forall s \in S, \\
& \sum_{p}\left(x l_{s p t q}+x o_{s p t q}\right) \leq C s_{s t q} y c_{s}, \quad \forall s \in S, t \in T,
\end{aligned}
$$$$
\sum_{p} x o_{s p t q} \leq \sum_{i} g o_{i} y o_{i s} \quad \forall s \in S, t \in T,
$$$$
\sum_{p} x s t_{p t q} \leq \sum_{j} \text { gst }_{j} y s t_{j} \quad \forall t \in T,
$$$$
\sum_{p} x l_{s p t q} \leq \sum_{k} g l_{k} y l_{k s} \quad \forall s \in S, t \in T,
$$$$
\sum_{s}\left(x l_{s p t q}+x o_{s p t q}\right)=\sum_{w} x p w_{p w t q} \quad \forall p \in P, t \in T,
$$$$
\operatorname{Inv}_{w t+1 q}=\operatorname{Inv}_{w t q}+\sum_{p} x p w_{p w t q}-\sum_{c} x w c_{w c t q}
$$$$
\forall w \in W, t \in T
$$

$\sum_{w} x w c_{w c t q}+z_{c t q} \geq d_{c t q}$

$$
\forall c \in C, t \in T, q \in Q,
$$




$$
\begin{aligned}
& \operatorname{Inv}_{w 0 q}=0 \quad \forall w \in W \\
& x s t_{p t q}, x o_{s p t q}, x l_{s p t q}, x p w_{p w t q}, x w c_{w c t q}, z_{c t q} \\
& \quad \geq 0, y s t_{j}, y o_{i s}, y c_{s}, y l_{k s} \in\{0,1\} .
\end{aligned}
$$

The variance of above estimator has been shown in Eq. (6):

$$
s_{u}^{2}=\frac{1}{N^{\prime}\left(N^{\prime}-1\right)} \sum_{n=1}^{N^{\prime}}\left(d^{T} \bar{y}+Q\left(\bar{y}, \xi_{n}\right)-\bar{u}\right)^{2} .
$$

This procedure continues until the gap between upper and lower bounds reaches an a priori determined level. This gap and its corresponding variance, as for our problem, appear in Eqs. (7) and (8):

$$
\begin{aligned}
& \mu_{g a p}=\bar{u}-\bar{l}, \\
& \sigma_{g a p}^{2}=s_{u}^{2}+s_{l}^{2} .
\end{aligned}
$$

To assess the quality of the solutions obtained so far, Santoso et al. [9] proposed to construct a confidence interval based on normal distribution, which is depicted in Eq. (9):

$$
\left[0, \mu_{g a p}+z_{\frac{\alpha}{2}} \sigma_{g a p}\right] .
$$

Although applying the SAA gives a good opportunity to reduce the size problem significantly, there is still a problem significantly, there is still a problem with the replications needed to solve this problem, that is, $M$. To further reduce the computational time required to address the size problem, one may need to resort to exploiting the structure of the problem. Since the problem under consideration is a two-stage stochastic program, Benders decomposition technique has been proposed for them in the literature [28] due to the complicating first-stage variables. In the next section, a brief description of this technique is illustrated.

\subsection{Benders decomposition}

In his paper, Benders [30] proposed a decomposition scheme for problems with complicating variables. He suggested that the problem decomposes into more tractable sub-problems by fixing these variables. Consider the problem in Relation (10) in which variables $y$ are complicating:

$$
\begin{aligned}
& \min _{y, x} d^{T} y+c^{T} x, \\
& \text { s.t.: } B y+A x \leq b, \\
& F y \leq p .
\end{aligned}
$$

Benders proved that by solving the problem in Relation (11), called Master problem, not only a fixed value for decision vector $y$ will be obtained, but also a lower bound for the main problem will be reached. In each iteration, this value results in the sub problem of Relation (12). Solving this sub problem has two benefits: constructing an upper bound along with new information from the main problem that can be passed through a set of new constraints (cuts) to the Master. The procedure continues until a suitable gap is attained.

$$
\begin{aligned}
& \min _{\theta, y} \theta, \\
& \text { s.t.: } F y \leq p, \\
& d^{T} y+u_{i}^{T}(b-B y) \leq \theta \rightarrow \text { Optimality cut } \\
& \quad \forall i \in \text { Constraints set, } \\
& \quad \forall i \in \text { Constraints set, } \\
& v_{i}^{T}(b-B y) \leq 0 \rightarrow \text { Feasibility cut } \\
& \min _{x} c^{T} x, \\
& \text { s.t.: } A x \leq b-B \bar{y} .
\end{aligned}
$$

As can be seen in Relations (11), there are two kinds of cuts. Feasibility cuts that enforce the Master problem to generate feasible solutions and optimality cuts that iteratively cut sub-optimal solutions from Master problem. It may be of interest to mention that in our model, the second-stage problem is always feasible for all configurations issuing from the firststage problem, since a term has been added to grasp unmet demand in Constraint (2-9). In this regard, the first-stage problem with $d^{T} y$ is denoted as in Eq. (13):

$$
\begin{aligned}
d^{T} y= & \sum_{s} F c_{s} y c_{s}+\sum_{j} F s t_{j} y s t_{j}+\sum_{i, s} F o_{i s} y o_{i s} \\
& +\sum_{k, s} F l_{k s} y l_{k s} .
\end{aligned}
$$

The Master problem could be rewritten as problem in Relation (14):

The Master problem:

$\min \theta$,

$$
\begin{aligned}
& \text { s.t.: }(1-1)-(1-4), \\
& y_{s t_{j}}, y o_{i s}, y c_{s}, y l_{k s} \in\{0,1\} .
\end{aligned}
$$

Benders cuts: 


$$
\begin{aligned}
\theta & \geq d^{T} y+\sum_{s, t, n} D_{s t n}^{21} C s_{s t n} y c_{s} \\
& +\sum_{s, t, n} D_{s t n}^{22}\left(\sum_{i} g o_{i} y o_{i s}\right)+\sum_{t, n} D_{t n}^{23}\left(\sum_{j} g s t_{j} y s t_{j}\right) \\
& +\sum_{s, t, n} D_{s t n}^{24}\left(\sum_{k} g l_{k} y l_{k s}\right)+\sum_{w, t, n} D_{w t n}^{27} C w_{w t n} \\
& +\sum_{c, t, n} D_{c t n}^{29} d_{c t n}
\end{aligned}
$$

where the notation $D_{a b c}^{p q}$ stands for the dual value related to constraint $p q$, while $a b c$ shows the counter for this constraint set. In addition, sub problem will look like the problem in Relation (15):

$$
\begin{aligned}
& \min _{x, z} q_{n}^{T} x_{n} \\
& \sum_{p}\left(x l_{s p t n}+x o_{s p t n}\right) \leq C s_{s t n} \overline{y c}_{s} \\
& \forall s \in S, t \in T, n \in N, \\
& \sum_{p} x o_{s p t n} \leq \sum_{i} g o_{i} \overline{y o}_{i s} \\
& \forall s \in S, t \in T, n \in N, \\
& \sum_{p} x s t_{p t n} \leq \sum_{j} g s t_{j} \overline{y s t}_{j} \quad \forall t \in T, n \in N, \\
& \sum_{p} x l_{s p t n} \leq \sum_{k} g l_{k} \overline{y l}_{k s} \quad \forall s \in S, t \in T, n \in N
\end{aligned}
$$

$$
x s t_{p t n}, x o_{s p t n}, x l_{s p t n}, x p w_{p w t n}, x w c_{w c t n}, z_{c t n} \geq 0 .
$$

\subsection{Acceleration schemes}

Many researchers have come to the conclusion that Benders decomposition in its basic format has poor convergence behavior [31]. To overcome this obstacle, in this paper, a bunch of acceleration techniques has been used. In the following, techniques that have been used in this paper will be described.

\subsubsection{Multi-cut version}

Birge and louveaux [32] observed that in each iteration of Benders decomposition applied to a twostage stochastic program, it is possible to generate a bunch of cuts up to the number of scenarios. Since these cuts constrain the Master problem, the time it takes to solve the Master may increase. However, since the feasible region becomes tighter, it is reasonable to think of better lower bounds for the main problem in each iteration. Thus, for problems with few scenarios, it may be worth a try using such a scheme. As SAA is used in this paper, which results in solving problems with fewer scenarios, there is a chance to implement this approach with the hope of increasing the convergence rate, as results approved it. In the classic Benders decomposition, the attempt is to approximate the objective function of the main problem, consisting of all scenarios, using variable $\theta$, as in Relations (11). However, in the multi-cut version, the idea is to approximate the objective function for each scenario. In this version, $\theta_{n}$ will approximate the objective function so that just one scenario is going to happen. Here, the Master problem takes the form of Relations (16):

$$
\begin{aligned}
& \min _{\theta_{n}, y} \sum_{n=1}^{N} \theta_{n}, \\
& \text { s.t.: } F y \leq p, \\
& d^{T} y+u_{n i}^{T}\left(b\left(\xi_{n}\right)-B y\right) \leq \theta_{n}, \\
& \quad \forall n \in n, \forall i \in \text { Constraints set. }
\end{aligned}
$$

\subsubsection{Valid inequalities}

Another important acceleration scheme in many applications is deriving constraints that do not change the feasible region of the original problem, but push the current constraints towards the facets of the problem. These constraints are called Valid Inequalities (VI). Adding these VIs to the Master initially results in better lower bounds, thus accelerates the convergence. To this end, many VIs have been derived based on the structure of the problem under consideration.

Proposition: The following inequalities are valid for the model described above:

$$
\begin{aligned}
& \sum_{t} \sum_{s} C s_{s t n} y c_{s} \geq \sum_{t} \sum_{c} d_{c t n}, \quad \forall n \in N, \\
& \sum_{t} \sum_{j} g s t_{j} y s t_{j} \geq \sum_{t} \sum_{c} d_{c t n}, \quad \forall n \in N, \\
& \sum_{t} \sum_{s}\left(\sum_{i} g o_{i} y o_{i}+\sum_{k} g l_{k} y l_{k}\right) \geq \sum_{t} \sum_{c} d_{c t n}
\end{aligned}
$$

$\forall n \in N$,

Constraints $(1-2),(1-3) \rightarrow \sum_{s} \sum_{i} y o_{i s} \leq \sum_{s} y c_{s} \leq \beta$,

Constraints $(1-4),(1-3) \rightarrow \sum_{s} \sum_{k} y l_{k s} \leq \sum_{s} y c_{s} \leq \beta$. 
Table 2. Characteristics of the case supply chain.

\begin{tabular}{lclc}
\hline \multicolumn{1}{c}{ Characteristic } & No. & \multicolumn{1}{c}{ Parameter } & Cost(\$) \\
\hline Customers & 2 & Warehouses & 2 \\
Plants & 2 & Long-term contract intervals & 6 \\
Non-strategic suppliers & 9 & Unit transportation cost & 1 \\
Capacity levels for non-strategic part & 5 & Inventory holding & 0.18 \\
Capacity levels for strategic part & 6 & Unit strategic part costs & 33 \\
Time periods & 52 & Mean total yearly demand & 903750 \\
Option levels for non-strategic parts & 5 & Mean warehouse capacity & 17380 \\
\hline
\end{tabular}

Table 3. Allowable purchasing levels for different contract types along with their fixed costs.

\begin{tabular}{cccccc}
\hline $\begin{array}{c}\text { Purchase } \\
\text { interval } \\
\text { ID }\end{array}$ & $\begin{array}{c}\text { Long-term } \\
\text { contract level } \\
\text { (Max./week) }\end{array}$ & $\begin{array}{c}\text { Capacity } \\
\text { reservation } \\
\text { level }\end{array}$ & $\begin{array}{c}\text { Option } \\
\text { contract level } \\
\text { (Max./week) }\end{array}$ & $\begin{array}{c}\text { Fixed cost to } \\
\text { engage with } \\
\text { strategic supplier (\$) }\end{array}$ & $\begin{array}{c}\text { Premium } \\
\text { option cost (\$) }\end{array}$ \\
\hline $\mathbf{1}$ & 2000 & 30000 & 400 & 40000 & 1200 \\
$\mathbf{2}$ & 3000 & 31500 & 2000 & 41500 & 2500 \\
$\mathbf{3}$ & 5000 & 33000 & 4000 & 43000 & 6190 \\
$\mathbf{4}$ & 7000 & 34500 & 6000 & 44500 & 15400 \\
$\mathbf{5}$ & 9000 & 36000 & 8000 & 47000 & 38300 \\
$\mathbf{6}$ & 11000 & - & - & - & - \\
\hline
\end{tabular}

\subsubsection{Trust region constraints}

One problem with Benders decomposition is the oscillation of the solutions found in each iteration from one side of the solution space to others. Authors, such as Linderoth and Wright [33], proposed to use the trust region method as a remedy. The idea of trust region method is to confine the amount to which, each variable can change in the next iteration, but as Santoso et al. [9] have pointed out, this method for the problems with binary variables changes as follows. In each iteration set, a bound on the number of binaries can take the value of 1 .

\subsubsection{Knapsack upper bounding technique}

Since the time Benders has introduced his work on decomposition, it was known that the lower bound on the optimal solution improves iteratively (or at least does not get worse). However, there is no guarantee on the upper bound. In this paper, knapsack inequalities are used, as introduced by Santoso [10], which are derived in the following way: Let $d^{T} y+u_{i}^{T}(b-B y)$ be the optimality cut of the $i$ th iteration and UB as the best upper bound found so far. Since it is needed to have $U B \geq \theta$, to control the upper bound in the next iteration, one may add $\left(d^{T}-u_{i}^{T} B\right) y \leq U B-u_{i}^{T} b$ as a new cut to the next Master problem.

\section{Computational results}

\subsection{Test problem generation}

As to test the abovementioned hybrid approach, in this paper, the case data from $\mathrm{Xu}$ and Nozick [26] is used.
However, since their model has been modified in this paper, new features should be added to theirs. In their case, the authors were looking for provisioning two plants with a single common part. These plants should jointly sign contracts with suppliers to ensure supply over the year. Each year is considered as 52 weeks, and these plants are going to feed two markets with their product. Other characteristics of this supply chain are illustrated in Tables 2 and 3 . Recall that in the process of SAA, it is needed to solve $M$ problems each with size $N$ to obtain a lower bound. Meanwhile, it is required to solve the problem with much bigger sample of size $N^{\prime}$ to achieve an upper bound on the true problem. In this case, 20, 60, and 2000 were used as these parameters to obtain a good quality solution. Also, the dimensions of the deterministic equivalent for this problem, with 60 scenarios, are depicted in Table 4 to show the size of the problems that are going to be solved $M$ times.

Since the assumption of certainty for several parameters has been relaxed in this paper, i.e. demand, outsourcing cost and supplier, and warehouse capaci-

Table 4. Dimensions of the deterministic equivalent.

\begin{tabular}{clccc}
\hline \multirow{2}{*}{$\mathbf{N}=\mathbf{6 0}$} & Type & $\begin{array}{c}\text { First } \\
\text { stage }\end{array}$ & $\begin{array}{c}\text { Second } \\
\text { stage }\end{array}$ & Total \\
\hline \multirow{2}{*}{ Constraints } & Inequality & 20 & 71760 & 112460 \\
& Equality & - & 40680 & \\
& Continuous & - & 156120 & 156240 \\
\hline
\end{tabular}


Table 5. Comparison of CPU time for $\mathrm{AB}$ and $\mathrm{Cplex}$ $(M=20)$.

\begin{tabular}{ccccc}
\hline & \multicolumn{2}{c}{ Benders } & & Cplex \\
\cline { 2 - 3 } & Iterations & $\begin{array}{c}\text { CPU } \\
\text { (seconds) }\end{array}$ & & $\begin{array}{c}\text { CPU } \\
\text { (seconds) }\end{array}$ \\
\hline $\mathbf{1 0}$ & 147 & 146.3 & & 149.2 \\
$\mathbf{2 0}$ & 139 & 264.4 & & 382.8 \\
$\mathbf{3 0}$ & 131 & 337.6 & & 777.2 \\
$\mathbf{4 0}$ & 136 & 479.0 & & 1365.4 \\
$\mathbf{5 0}$ & 126 & 546.0 & & 2100.4 \\
$\mathbf{6 0}$ & 132 & 753.4 & & 2965.0 \\
\hline
\end{tabular}

ties, in contrast to $\mathrm{Xu}$ and Nozick [26], deterministic values were used as mean value for the stochastic parameters. Also, the standard deviations of these distributions were considered as a percent of their mean values. The distribution used for these parameters is log normal except for the outsourcing price (since it is much unexpected that the price of a finished product goes to zero).

\subsection{Performance of acceleration schemes}

All experiments were run using $\mathrm{C \#}$ on the Visual Studio 2010 platform in conjunction with the $\mathrm{Cplex}^{\circledR}$. In Table 5, solution time in CPU seconds is compared between the accelerated approach and the Cplex solver. One point here is that as $\mathrm{Xu}$ and Nozick [26] reported, convergence time for this problem in the pure Benders type is very slow, even slower than Cplex. This should be added to the observation that the case considered here just involves two customers, two warehouses, and two plants. However, to be more realistic, at least the number of customers, if not simplified too much, would be far more. So, the use of the Accelerated Benders (AB) algorithm is justifiable. Figure 2 exhibits the behavior of different acceleration mixtures. However, the best performance is obtained from hybridization of knapsack cuts with valid inequalities.

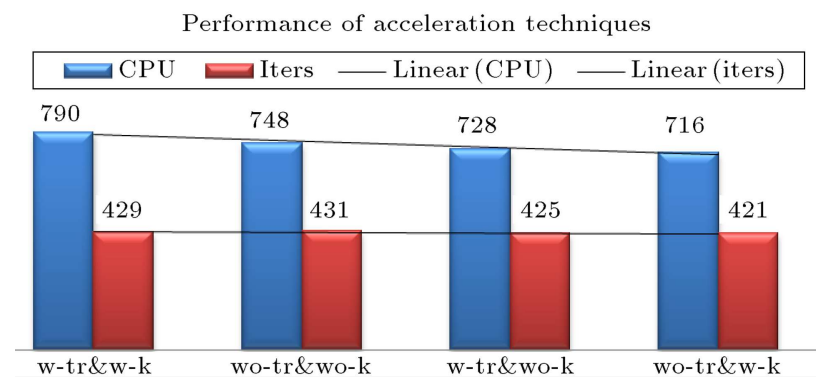

Figure 2. Comparison among acceleration techniques (w $=$ with, wo $=$ without, $\mathrm{tr}=$ trust region, $\mathrm{k}=$ knapsack $)$.

\subsection{Quality of the stochastic solutions}

This section is devoted to the comparison between stochastic and deterministic solutions from the aspect of solution quality. Here, by quality, it is meant centrality and diversity of the cost distribution that is given rise to by solutions from solving the twostage model or its deterministic counterpart. The distribution of costs for $M=20, N=60$, and $N^{\prime}=$ 2000 in both the stochastic problem and MVP (Mean Value Problem, since mean of stochastic parameters have been used as their sole value) is depicted in Figure 3. Also, in Table 6, parametric results regarding the comparison between the stochastic model and its deterministic equivalent are shown. These models have been solved using SAA-Benders algorithm with $N$ being set to 30,40 , and 60 . From all indexes of the SAA with 60 scenarios, it could be observed that stochastic solution is in a better situation. To illustrate, mean values for the total cost is lower than the cost associated with the MVP. Moreover, the standard deviation of the attained solution reveals much better performance. Row 2 of Table 6 shows that the most probable scenario with the stochastic solution has lower costs than the deterministic solution. Row 5 of this table shows a better opportunity for having low costs if using the stochastic solution. In addition, Row 6 shows a signal that the worst possible case related to the

Table 6. Cost statistics for stochastic and deterministic solutions.

\begin{tabular}{lcccc}
\hline \multirow{2}{*}{ Measure } & \multicolumn{4}{c}{ Solution } \\
\cline { 2 - 5 } & SAA & SAA & SAA & MVP \\
\hline Mean & $(\boldsymbol{N}=\mathbf{3 0})$ & $(\boldsymbol{N}=\mathbf{4 0})$ & $(\boldsymbol{N}=\mathbf{6 0})$ & \\
Mode & $3.79 \mathrm{E}+08$ & $3.54 \mathrm{E}+08$ & $\mathbf{3 . 5 E}+\mathbf{0 8}$ & $\mathbf{3 . 9 8 E + 0 8}$ \\
Standard deviation & $3.45 \mathrm{E}+08$ & $3.58 \mathrm{E}+08$ & $\mathbf{3 . 4 E}+\mathbf{0 8}$ & $\mathbf{3 . 6 3 E}+\mathbf{0 8}$ \\
Range & $4.2 \mathrm{E}+07$ & $1.4 \mathrm{E}+07$ & $\mathbf{1 . 1 E}+\mathbf{0 7}$ & $\mathbf{2 . 8 E}+\mathbf{0 7}$ \\
Minimum & $16.1 \mathrm{E}+07$ & $7.2 \mathrm{E}+07$ & $\mathbf{5 . 4 E}+\mathbf{0 7}$ & $\mathbf{9 . 1 E}+\mathbf{0 7}$ \\
Maximum & $3.16 \mathrm{E}+08$ & $3.3 \mathrm{E}+08$ & $\mathbf{3 . 3 E}+\mathbf{0 8}$ & $\mathbf{3 . 6 E}+\mathbf{0 8}$ \\
Gap (in million $\$)$ & $4.8 \mathrm{E}+08$ & $4 \mathrm{E}+08$ & $\mathbf{3 . 8 E}+\mathbf{0 8}$ & $\mathbf{4 . 5 E}+\mathbf{0 8}$ \\
$\boldsymbol{\sigma}$ & 20.34 & 2.39 & $\mathbf{0 . 1 1}$ & $\mathbf{4 8 . 2 7}$ \\
Confidence level $(\mathbf{9 5 . 0 \% )}$ & 2613527 & 855107 & 683545 & 1722400 \\
\hline
\end{tabular}



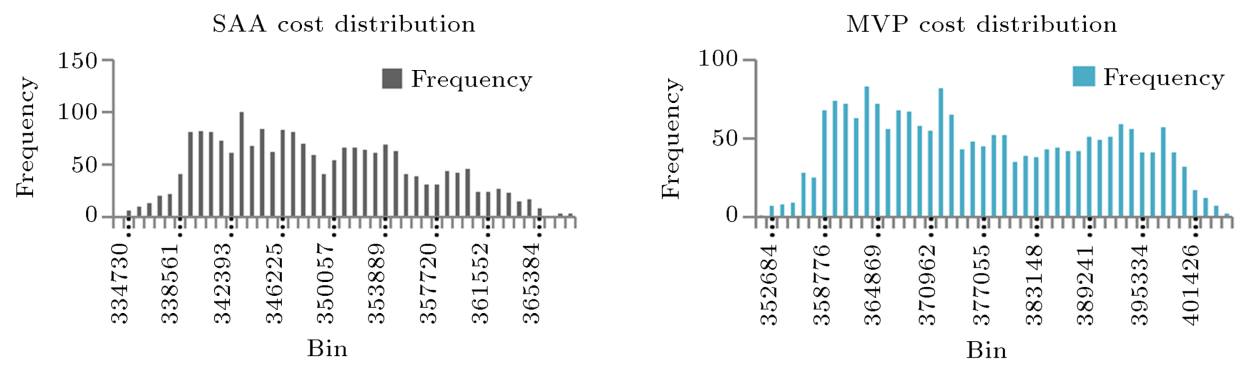

Figure 3. Total cost histogram for stochastic and deterministic solutions $\left(M=20, N=60\right.$, and $\left.N^{\prime}=2000\right)$.

stochastic solution is also much less. Finally, Row 7 Gap and its standard deviation exhibit lower figures, which guarantee the probability that the solution for the SAA problem being close to the real solution is higher than with MVP. Comparing these statistics for SAA with different number of scenarios, one can see that in almost all of these statistics, especially the worst case costs, quality of the stochastic solution is getting better with the increase in sample sizes with regard to mean and standard deviation of the total costs and corresponding values for SAA gap. As a final point, the last row of Table 6 gives the second term in Relation 9 to obtain a $95 \%$ confidence interval on the optimality gap.

As for the last step, the results were examined by changing variability of the stochastic parameters. Up to now, results have been based on the same amount of variability in uncertain parameters. However, in this section, these values are changed according to the categories in Table 7 . In this table, each row shows the percentage of standard deviation (sd) of the uncertain parameter with respect to its mean value. Figure 4 exhibits that change of variability enhances the Value of Stochastic Solutions (VSS, which shows the difference between the cost of the system with deterministic parameters and the cost of the system

Table 7. Different variability categories for stochastic parameters.

\begin{tabular}{lccc}
\hline \multicolumn{1}{c}{ Category } & Low & Medium & High \\
\hline Demand sd\% & 20 & 30 & 40 \\
Others sd\% & 5 & 10 & 15 \\
\hline
\end{tabular}
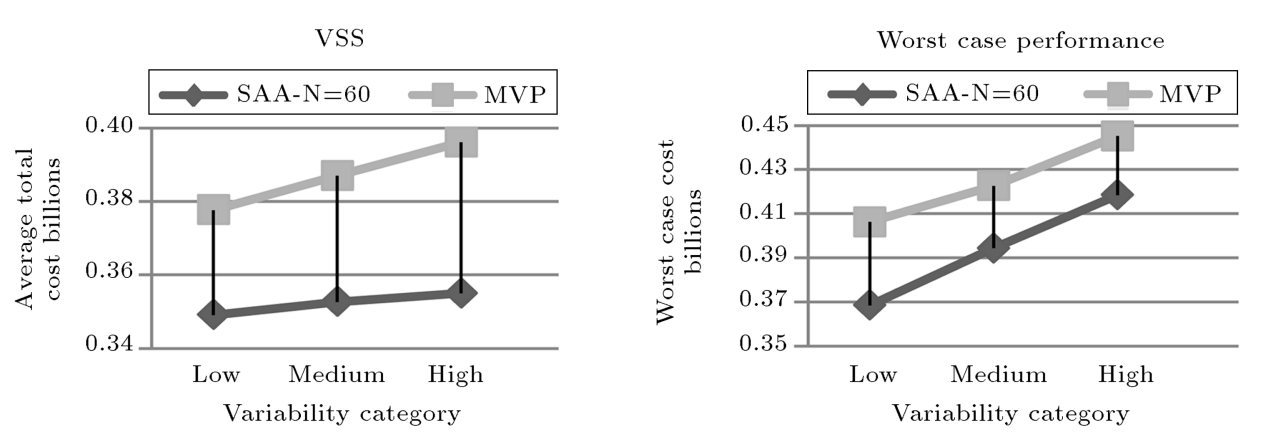
counterparts in an obvious manner. In other words, our solutions are more robust considering that the environment is changing. Although the results for the worst case cost show a better performance for stochastic solution, but they do not show the same robustness as VSS. However, since the mean and the worst case costs do not show a good measure of risk as argued by many researchers (mean ignores too many of the possible events and the worst case cost is a rare event), it is preferred to use Value at Risk (VaR) and Conditional Value at Risk (CVaR), which is the rather recently introduced risk measure, to compare solutions in different categories of variability. VaR metric considers risky events up to $95 \%$ of the cost distributions, and CVaR even measures the mean of events passing VaR too. As illustrated in Figure 5, it could be seen that even for these two measures, the stochastic solution shows a better performance in different variability categories.

\section{Conclusions and future research directions}

In this paper, for the first time, different contracts for strategic and non-strategic items are incorporated into supply chain network design problem. Also, as mentioned before, this paper is one of the few papers considering stochastic uncertainty in most of the parameters related to the field of supply chain network design. This could be conceived as a new research area, and other strategic contracts, such as buyback, revenue sharing, etc. could be incorporated in the model according to the situation. Results of the

Figure 4. Comparison of average total cost/VSS and the worst total cost for different variability categories. 

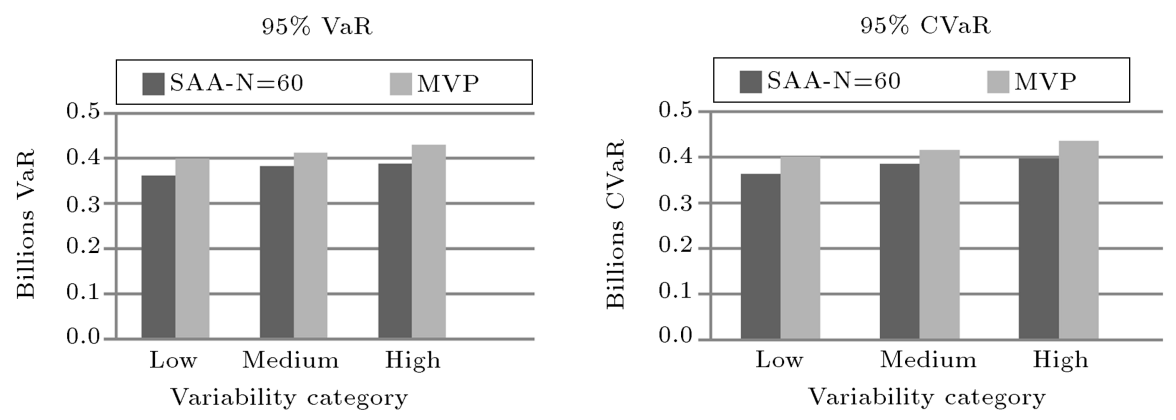

Figure 5. Comparison of VaR and CVaR measures between stochastic and deterministic solutions.

proposed model show that, in many ways, stochastic solution has priority over the mean value solution, and the usage of stochastic model will result in supply chain designs with high cost savings as compared to traditional deterministic models. The robustness and value of the stochastic model are also shown to increase with uncertainty levels. To address the solution of the proposed model, a hybrid strategy based on sample average approximation and an accelerated version of the Benders decomposition is used. This algorithm is able to handle large sets of scenarios or even continuous distributions. Thus, the proposed hybrid solution strategy is eligible to be used for real life problem sizes. This algorithm could be tuned such that the lowest sample size needed to obtain a good quality solution is attained. In addition, in this paper, a modified version of the multi-cut Benders decomposition is used according to the structure of the problem at hand, including introducing new valid inequalities, such that the proposed algorithm outperforms traditional Benders. Another advantage of our model and the proposed solution strategy is that it can easily grasp uncertainties in other parameters such as transportation fees, manufacturer's capacity, and inventory holding cost. Also, a possible extension is to minimize a risk measure such as CVaR instead of minimizing expected value cost to achieve more robust solutions.

\section{References}

1. Simchi-Levi, D., Kaminsky, P. and Simchi-Levi, E., Designing and Managing the Supply Chain: Concepts, Strategies, and Case Studies, McGraw-Hill/Irwin (2007).

2. Kraljic, P. "Purchasing must become supply management", Harvard Business Review, 61, pp. 109-117 (1983).

3. Cachon, G.P. and Lariviere, M.A. "Contracting to assure supply: How to share demand forecasts in a supply chain", Manage. Sci., 47, pp. 629-646 (2001).

4. Özer, Ö. and Wei, W. "Strategic commitments for an optimal capacity decision under asymmetric forecast information", Management Science, 52, pp. 1238-1257 (2006).
5. Thanh, P.N., Péton, O. and Bostel, N. "A linear relaxation-based heuristic approach for logistics network design", Computers \& Industrial Engineering, 59, pp. 964-975 (2010).

6. Nagurney, A. "Optimal supply chain network design and redesign at minimal total cost and with demand satisfaction", International Journal of Production Economics, 128, pp. 200-208 (2010).

7. Badri, H., Bashiri, M. and Hejazi, T.H. "Integrated strategic and tactical planning in a supply chain network design with a heuristic solution method", Computers \& Operations Research, 40, pp. 1143-1154 (2013).

8. Correia, I., Melo, T. and Saldanha-da-Gama, F. "Comparing classical performance measures for a multiperiod, two-echelon supply chain network design problem with sizing decisions", Computers \& Industrial Engineering, 64, pp. 366-380 (2013).

9. Santoso, F., Ahmed, S., Goetschalckx, M. and Shapiro, A. "A stochastic programming approach for supply chain network design under uncertainty", European Journal of Operational Research, 167, pp. 96-115 (2005).

10. Santoso, T., A Comprehensive Model and Efficient Solution Algorithm for the Design of Global Supply Chains Under Uncertainty, School of Industrial and Systems Engineering, Georgia Institute of Technology, Directed by Marc Goetschalckx (2003).

11. Tiwari, M.K., Raghavendra, N., Agrawal, S. and Goyal, S.K. "A hybrid Taguchi-immune approach to optimize an integrated supply chain design problem with multiple shipping", European Journal of Operational Research, 203, pp. 95-106 (2010).

12. Pan, F. and Nagi, R. "Robust supply chain design under uncertain demand in agile manufacturing", Computers \& Operations Research, 37, pp. 668-683 (2010).

13. Georgiadis, M.C., Tsiakis, P., Longinidis, P. and Sofioglou, M.K. "Optimal design of supply chain networks under uncertain transient demand variations", Omega, 39, pp. 254-272 (2011).

14. Rajgopal, J., Wang, Z., Schaefer, A.J. and Prokopyev, O.A. "Integrated design and operation of remnant inventory supply chains under uncertainty", European Journal of Operational Research, 214, pp. 358-364 (2011). 
15. Sawik, T. "Selection of supply portfolio under disruption risks", Omega, 39, pp. 194-208 (2011).

16. Li, L. and Zabinsky, Z.B. "Incorporating uncertainty into a supplier selection problem", International Journal of Production Economics, 134, pp. 344-356 (2011).

17. Lin, C.-C. and Wang, T.-H. "Build-to-order supply chain network design under supply and demand uncertainties", Transportation Research Part B: Methodological, 45, pp. 1162-1176 (2011).

18. Pishvaee, T.-H. and Razmi, J. "Environmental supply chain network design using multi-objective fuzzy mathematical programming", Applied Mathematical Modelling, 36, pp. 3433-3446 (2012).

19. Goetschalckx, M., Huang, E. and Mital, P. "Trading off supply chain risk and efficiency through supply chain design", Procedia Computer Science, 16, pp. 658-667 (2013).

20. Tabrizi, B.H. and Razmi, J. "Introducing a mixedinteger non-linear fuzzy model for risk management in designing supply chain networks", Journal of Manufacturing Systems, 32, pp. 295-307 (2013).

21. Pishvaee, M.S., Razmi, J. and Torabi, S.A. "An accelerated Benders decomposition algorithm for sustainable supply chain network design under uncertainty: A case study of medical needle and syringe supply chain", Transportation Research Part E: Logistics and Transportation Review, 67, pp. 14-38 (2014).

22. Yolmeh, A. and Salehi, N. "An outer approximation method for an integration of supply chain network designing and assembly line balancing under uncertainty", Computers \& Industrial Engineering, 83, pp. 297-306 (2015).

23. Wang, K.-J. and Lee, C.H. "A revised ant algorithm for solving location-allocation problem with risky demand in a multi-echelon supply chain network", Applied Soft Computing, 32, pp. 311-321 (2015).

24. Melo, M.T., Nickel, S. and Saldanha-da-Gama, F. "Facility location and supply chain management - A review", European Journal of Operational Research, 196, pp. 401-412 (2009).

25. Paksoy, T., Özceylan, E. and Weber, G.-W. "Profit oriented supply chain network optimization", Cent. Eur. J. Oper. Res., 21, pp. 455-478 (2013).

26. Xu, N. and Nozick, L. "Modeling supplier selection and the use of option contracts for global supply chain design", Computers \& Operations Research, 36, pp. 2786-2800 (2009).

27. Feng, Y., Martel, A., D'Amours, S. and Beauregard, R. "Coordinated contract decisions in a maketo-order manufacturing supply chain: A stochastic programming approach", Production and Operations Management, 22, pp. 642-660 (2013).

28. Van Slyke, R. and Wets, R. "L-shaped linear programs with applications to optimal control and stochastic programming", SIAM Journal on Applied Mathematics, 17, pp. 638-663 (1969).
29. Shapiro, A. and Homem-de-Mello, T. "A simulationbased approach to two-stage stochastic programming with recourse", Mathematical Programming, 81, pp. 301-325 (1998).

30. Benders, J.F. "Partitioning procedures for solving mixed-variables programming problems", Numer. Math., 4, pp. 238-252 (1962).

31. Azad, N., Saharidis, G.D., Davoudpour, H., Malekly, H. and Yektamaram, S. "Strategies for protecting supply chain networks against facility and transportation disruptions: an improved Benders decomposition approach", Ann Oper. Res., 210, pp. 125-163 (2013).

32. Birge, J.R. and Louveaux, F.V. "A multicut algorithm for two-stage stochastic linear programs", European Journal of Operational Research, 34, pp. 384-392 (1988).

33. Linderoth, J. and Wright, S. "Decomposition algorithms for stochastic programming on a computational grid", Computational Optimization and Applications, 24, pp. 207-250 (2003).

\section{Appendix}

Before proving the proposition, a lemma that helps with the proof of valid inequalities will be addressed:

Lemma: The following relations are correct based on the feasible region of the second stage problem:

$$
\begin{aligned}
& \sum_{w} x w c_{w c t n} \geq d_{c t n} \\
& \operatorname{Inv}_{w T n}=\sum_{t=0}^{T-1}\left(\sum_{p} x p w_{p w t n}-\sum_{c} x w c_{w c t n}\right) \\
& \sum_{t} \sum_{p} \sum_{w} x p w_{p w t n} \geq \sum_{t} \sum_{c} d_{c t n} .
\end{aligned}
$$

Proof of the Lemma: The first inequality (Relation (A.1)) is easily derived from Constraint (2-10). For the case of equality, (Eq. (A.2)) note that:

$$
\begin{aligned}
&(2-8) \rightarrow \operatorname{Inv}_{w t+1 n}= \operatorname{Inv}_{w t n}+\sum_{p} x p w_{p w t n} \\
&-\sum_{c} x w c_{w c t n} \\
& \operatorname{Inv}_{w t n}=\operatorname{Inv}_{w t-1 n}+\sum_{p} x p w_{p w t-1 n}-\sum_{c} x w c_{w c t-1 n} \\
& \vdots \\
& \operatorname{Inv}_{w 2 n}=\operatorname{Inv}_{w 1 n}+\sum_{p} x p w_{p w 1 n}-\sum_{c} x w c_{w c 1 n}
\end{aligned}
$$




$$
\begin{aligned}
& \operatorname{Inv}_{w 1 n}=\operatorname{Inv}_{w 0 n}+\sum_{p} x p w_{p w 0 n}-\sum_{c} x w c_{w c 0 n} \\
& (2-10): \operatorname{Inv}_{w 0 n}=0 \Rightarrow \operatorname{Inv}_{w 1 n}=\sum_{p} x p w_{p w 0 n} \\
& -\sum_{c} x w c_{w c 0 n} \\
& \Rightarrow \operatorname{Inv}_{w 2 n}=\sum_{p} x p w_{p w 0 n}-\sum_{c} x w c_{w c 0 n} \\
& +\sum_{p} x p w_{p w 1 n}-\sum_{c} x w c_{w c 1 n} \\
& \operatorname{Inv}_{w t n}=\sum_{p} x p w_{p w 0 n}-\sum_{c} x w c_{w c 0 n}+\sum_{p} x p w_{p w 1 n} \\
& -\sum_{c} x w c_{w c 1 n}+\cdots+\sum_{p} x p w_{p w t-1 n} \\
& -\sum_{c} x w c_{w c t-1 n} \\
& \Rightarrow \operatorname{Inv}_{w T n}=\sum_{t=0}^{T-1}\left(\sum_{p} x p w_{p w t n}-\sum_{c} x w c_{w c t n}\right) .
\end{aligned}
$$

Finally, for Relation (A.3) we have the following:

$$
\begin{aligned}
& \operatorname{Inv}_{w T n} \geq 0 \rightarrow \sum_{t=0}^{T-1}\left(\sum_{p} x p w_{p w t n}-\sum_{c} x w c_{w c t n}\right) \geq 0, \\
& \sum_{t=0}^{T-1}\left(\sum_{c} x w c_{w c t n}\right) \leq \sum_{t=0}^{T-1}\left(\sum_{p} x p w_{p w t n}\right) \\
& \quad \stackrel{\sum_{w} T-1}{\rightarrow} \sum_{t=0} \sum_{c} \sum_{w} x w c_{w c t n} \leq \sum_{t=0}^{T-1} \sum_{p} \sum_{w} x p w_{p w t n}, \\
& \sum_{t=0}^{T-1} \sum_{c} \sum_{w} x w c_{w c t n} \stackrel{\sum_{t} \sum_{c}}{\longrightarrow} \geq \sum_{t=0}^{T-1} \sum_{c} d_{c t n}, \\
& (\mathrm{~A} .4),(\mathrm{A} .5) \rightarrow \sum_{t} \sum_{p} \sum_{w} x p w_{p w t n} \geq \sum_{t} \sum_{c} d_{c t n} .
\end{aligned}
$$

Proof. For the case of the first valid equality set (Eq. (17)), consider the following:

$$
\begin{gathered}
(2-5) \Rightarrow \sum_{t} \sum_{p} \sum_{s}\left(x l_{s p t n}+x o_{s p t n}\right) \\
=\sum_{t} \sum_{p} \sum_{w} x p w_{p w t n},
\end{gathered}
$$

$$
\begin{aligned}
& (2-1) \Rightarrow \sum_{t} \sum_{s} \sum_{p}\left(x l_{s p t n}+x o_{s p t n}\right) \\
& \quad \leq \sum_{t} \sum_{s} C s_{s t n} \overline{y c_{s}}, \\
& (\mathrm{~A} .3),(\mathrm{A} .6),(\mathrm{A} .7) \rightarrow \sum_{t} \sum_{s} C s_{s t n} \overline{y c_{s}} \geq \sum_{t} \sum_{c} d_{c t n},
\end{aligned}
$$

$\forall n \in N$.

The second valid inequality (Eq. (18)) proves as follows:

$$
\begin{aligned}
& (2-6)+(\mathrm{A} .3) \Rightarrow \sum_{t} \sum_{p} x s t_{p t n} \geq \sum_{t} \sum_{c} d_{c t n}, \\
& (2-3) \rightarrow \sum_{t} \sum_{p} x s t_{p t n} \leq \sum_{t} \sum_{j} g s t_{j} \overline{y s t_{j}} \\
& (\mathrm{~A} .8),(\mathrm{A} .9) \rightarrow \sum_{t} \sum_{j} g s t_{j} \overline{y s t_{j}} \geq \sum_{t} \sum_{c} d_{c t n}
\end{aligned}
$$

$\forall n \in N$.

And, the third valid inequality is proved in this way:

$$
\begin{gathered}
(2-2)+(2-4) \rightarrow \sum_{t} \sum_{s} \sum_{p}\left(x l_{s p t n}+x o_{s p t n}\right) \\
\leq \sum_{t} \sum_{s}\left(\sum_{i} g o_{i} \overline{y o_{i}}+\sum_{k} g l_{k} \overline{y l_{k}}\right), \quad(\mathrm{A} .10) \\
(\mathrm{A} .3),(\mathrm{A} .7),(\mathrm{A} .10) \rightarrow \sum_{t} \sum_{s}\left(\sum_{i} g o_{i} \overline{y o_{i}}+\sum_{k} g l_{k} \overline{y l_{k}}\right) \\
\geq \sum_{t} \sum_{c} d_{c t n}, \quad \forall n \in N .
\end{gathered}
$$

\section{Biographies}

Mohammad Mohajer Tabrizi is currently a $\mathrm{PhD}$ candidate of Industrial Dngineering in Amirkabir University of Technology, Department of Industrial Engineering and Management Systems. He received his BS from K.N. Toosi University of Technology and his MS from Shahed University, Tehran, Iran. His research focuses on supply chain management, contract management, stochastic programming, decomposition algorithms, and theory of constraints. He is currently working on effectively using contracts as hedging mechanisms in the field of supply chain network design under uncertainty, especially by means of recent powerful risk-averse stochastic programming techniques.

Behrooz Karimi is currently a Professor of Faculty of Industrial Engineering \& Management Systems in 
Amirkabir University of Technology. He received his $\mathrm{PhD}$ in Industrial Engineering from Amirkabir University of Technology, Tehran, Iran in 2002. His main research interests are logistics and supply chain management and applications of meta-heuristics.

Seyyed Ali Mirhassani is an Associate Professor in Amirkabir University of Technology, Department of
Mathematics. He received his PhD in Applied Mathematics (Operations Research) from Brunel University, UK in 1989, an MSc and BSc in Mathematics from Mashhad University, Mashhad, Iran. His research focuses on Combinatorial Optimization, Modelling, and Stochastic Programming. He has published one book, 3 book chapters, about 90 papers in technical journals, and more than 60 papers in conference proceedings. 